\title{
Interfaces et fluides critiques
}

\section{Interfaces and critical fluids}

\section{Roger Prud'homme ${ }^{1}$}

${ }^{1}$ Directeur de Recherche Emérite - Institut Jean Le Rond D’Alembert - Université Pierre et Marie Curie/CNRS

RÉSUMÉ. Pour les corps purs, l'interface de séparation de phases liquide-vapeur disparait au point critique. Cette propriété et d'autres encore ont suscité l'intérêt de nombreux scientifiques. Cet article est une mise à jour du texte de la conférence invitée sur les Fluides Critiques présentée à un séminaire de Saint-Gobain-Recherche Aubervilliers le 14 juin 2007. Sont présentés successivement : la thermostatique, puis la thermodynamique des fluides au voisinage du point critique ; l'effet piston, mode spécifique de transmission de la chaleur ; l'expansion d'une "goutte" a la pression critique ; le comportement d'une poche de fluide supercritique plongée dans un environnement à haute température ; enfin l'ébullition près du point critique. II s'agit pour partie d'une reprise de textes émis par différents auteurs cités.

ABSTRACT. For pure substances, the liquid-vapor phase separation interface disappears at the critical point. This property and others have attracted the interest of many scientists. This article is an update of the text of the invited conference on Critical Fluids presented at a seminar of Saint-Gobain-Recherche Aubervilliers on June 14, 2007. Thermostatics is first presented; then successively the thermodynamics of fluids near the critical point; the piston effect, a specific mode of heat transfer; the expansion of a "drop" at the critical pressure; the behavior of a supercritical fluid pocket immersed in a high temperature environment; finally boiling near the critical point. It is partly a resumption of texts issued by various authors cited.

MOTS-CLÉS. interface, fluide critique, phénomènes de transfert, changement de phase liquide-vapeur, évaporation, micro pesanteur, fluide supercritique.

KEYWORDS. interface, critical fluid, transfer phenomena, liquid-vapor phase change, evaporation, micro gravity, supercritical fluid.

\section{Liste des symboles}

\begin{tabular}{|c|c|c|c|}
\hline Symbole & Signification & Symbole & Signification \\
\hline$a, b$ & $\begin{array}{c}\text { Constantes de Van } \\
\text { der Waals }\end{array}$ & $\alpha, \beta, \gamma, \delta, v$ & $\begin{array}{l}\text { Exposants du groupe } \\
\text { de renormalisation }\end{array}$ \\
\hline$D$ & Diamètre de goutte & $\kappa=\lambda / \rho c_{p}$ & Diffusivité thermique \\
\hline$c_{p}$ & $\begin{array}{l}\text { Chaleur spécifique à } \\
\text { pression constante }\end{array}$ & $\lambda$ & $\begin{array}{l}\text { Conductivité } \\
\text { thermique }\end{array}$ \\
\hline$F, \bar{F}$ & $\begin{array}{c}\text { Energie libre, énergie } \\
\text { libre molaire }\end{array}$ & $\mu$ & $\begin{array}{l}\text { Viscosité de } \\
\text { cisaillement }\end{array}$ \\
\hline$K_{T}=(\partial \ln \rho / \partial p)_{T}$ & $\begin{array}{l}\text { Compressibilité } \\
\text { isotherme }\end{array}$ & $\rho, \rho_{\mathrm{c}}$ & $\begin{array}{l}\text { Masse volumique, } \\
\text { masse volumique } \\
\text { critique }\end{array}$ \\
\hline$p, p_{c}$ & $\begin{array}{l}\text { Pression, pression } \\
\text { critique }\end{array}$ & $\sigma$ & Tension de surface \\
\hline$R$ & $\begin{array}{l}\text { Constante molaire } \\
\text { des gaz parfaits }\end{array}$ & $\xi$ & $\begin{array}{c}\text { Longueur de } \\
\text { corrélation }\end{array}$ \\
\hline$S$ & Entropie & $\tau$ & Temps adimensionnel \\
\hline$T, T_{c}$ & $\begin{array}{l}\text { Température en }{ }^{\circ} \mathrm{K} \text {, } \\
\text { température critique }\end{array}$ & $v, \bar{v}$ & $\begin{array}{c}\text { Volume, volume } \\
\text { molaire }\end{array}$ \\
\hline
\end{tabular}

\footnotetext{
${ }^{1}$ Sorbonne Université, CNRS, Institut Jean Le Rond d'Alembert, UMR 7190, F-75005 Paris, France - e-mail : roger.prud_homme@upmc.fr
} 
La distinction entre les différentes phases d'un corps pur est généralement assez aisée, mais il peut se trouver des situations plus difficiles. Par exemple, pour un fluide pur sous pression suffisante, le ménisque habituel séparant le liquide de la phase gazeuse peut disparaître et laisser la place à une réelle continuité entre ces deux phases. C'est l'état de fluide supercritique (figure 1).

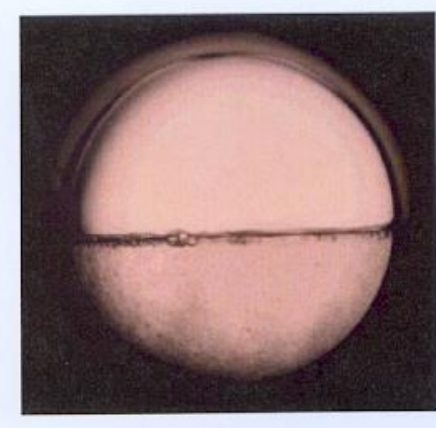

(a)

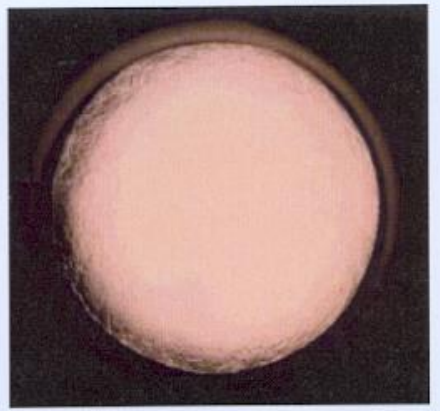

(b)

Figure 1. (a) les 2 phases (liquide et gaz) clairement apparentes (ménisque) pour le $\mathrm{CO}_{2}$, b) avec un accroissement de température, lorsque la température et la pression critiques sont atteintes, on ne distingue plus 2 phases (liquide et gaz), il n'y a plus qu'une seule phase : le fluide supercritique (Laboratoire LCSG, Université de Leeds).

Le thème des fluides critiques et supercritiques (SCF pour "Supercritical Fluids") présente un intérêt fondamental et les applications potentielles des fluides supercritiques touchent un vaste domaine allant de l'industrie des lanceurs spatiaux au traitement des déchets organiques ${ }^{\mathrm{ii}}$.

Les fluides supercritiques, très compressibles et dilatables au voisinage du point critique, mais aussi très denses, sont sensibles à la pesanteur, de sorte que l'expérimentation en microgravité est souvent nécessaire pour en comprendre le comportement et les modéliser. D'autre part, au point critique, certaines propriétés physiques divergent (telles la compressibilité isotherme $K_{T}=(\partial \ln \rho / \partial p)_{T}$, la chaleur spécifique $c_{p}$ à pression constante et la conductivité thermique $\lambda$ ) ou tendent vers zéro (telle la diffusivité thermique $\kappa=\lambda / \rho c_{p}$, la transmission de la chaleur par conduction est considérablement freinée, c'est le "ralentissement critique"), et des phénomènes nouveaux apparaissent. Des expériences réalisées en micropesanteur ont permis de confirmer l'existence de "l'effet piston", qui est un nouveau mode de transmission de la chaleur mis en évidence par la théorie ${ }^{\mathrm{iii}}$.

\section{Thermostatique des fluides au voisinage du point critique}

Les éléments ci-dessous concernent les lois d'état des fluides réels. On connait assez bien les gaz parfaits, c'est-à-dire les gaz sous basses pressions. En revanche les comportements des gaz sous hautes pressions sont plus mal connus. Nous verrons cependant qu'il est possible d'utiliser le comportement de gaz parfait d'un fluide pour trouver la forme de ses lois d 'état aux pressions plus élevées.

\subsection{Fluides réels}

Le diagramme d'état d'un corps pur est schématiquement représenté sur la figure 2. Le domaine des gaz parfaits correspond aux faibles pressions et grands volumes. Au point critique liquide vapeur $\mathrm{C}$, les dérivées partielles première et seconde de la pression par rapport au volume à température constante s'annulent :

$(\partial p / \partial \overline{\mathcal{V}})_{T}=0, \quad\left(\partial^{2} p / \partial \overline{\mathcal{V}}^{2}\right)_{T}=0$ 


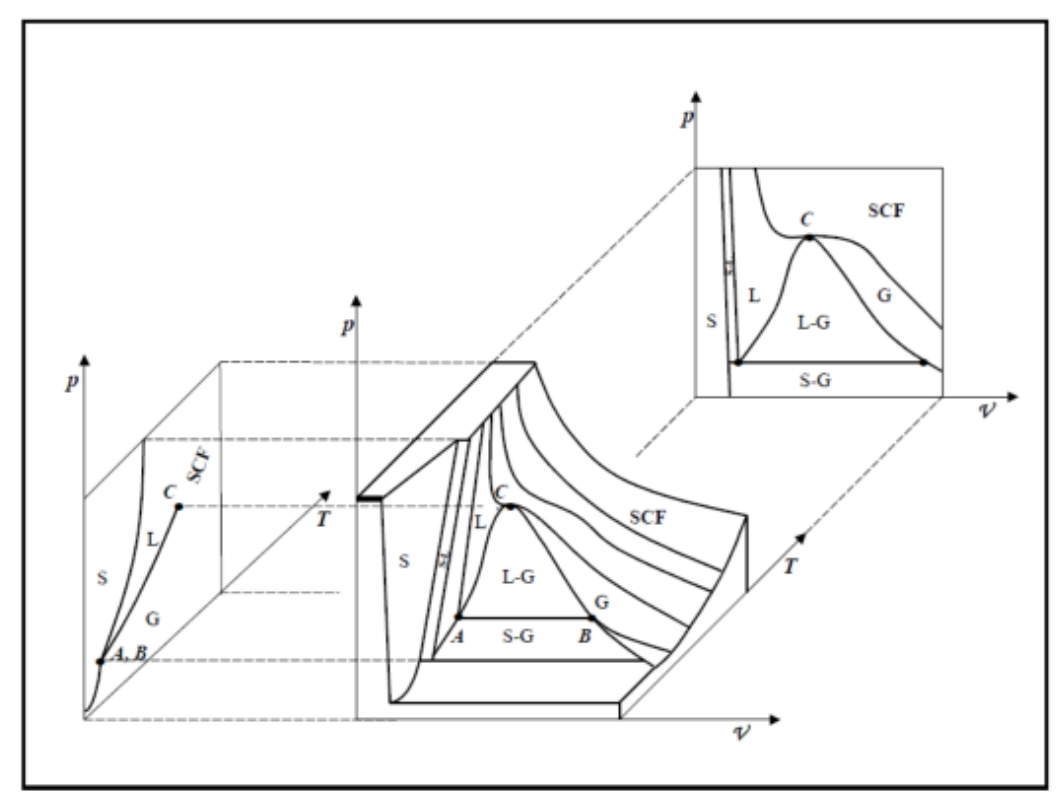

Figure 2. Diagramme d'état d'un corps pur.

$S$ : état solide, $L$ : état liquide, $G$ : état gazeux, SCF : fluide supercritique

Le tableau 1 donne les coordonnées du point critique de quelques corps ${ }^{\mathrm{iv}}$.

\begin{tabular}{|c|c|c|c|}
\hline Corps & $\begin{array}{c}\text { Température } \\
\text { Critique } \mathbf{T}_{\mathbf{c}}(\mathbf{K})\end{array}$ & $\begin{array}{c}\text { Pression } \\
\text { Critique } \mathbf{p}_{c}(\mathbf{M P a})\end{array}$ & $\begin{array}{l}\text { Masse volumique } \\
\text { critique }\left(\mathrm{Kg} / \mathrm{m}^{3}\right)\end{array}$ \\
\hline Eau & 647,1 & 22,06 & 322,2 \\
\hline Dioxyde de carbone & 304,14 & 7,378 & 467,8 \\
\hline Oxygène & 154,58 & 5,043 & 436,2 \\
\hline Azote & 126,24 & 3,398 & 313,9 \\
\hline Ammoniac & 405,4 & 11,1 & 235 \\
\hline Hélium & 3,316 & 0,114 & 41,45 \\
\hline
\end{tabular}

Tableau 1. Coordonnées du point critique de quelques corps [Garrabos 1992].

La loi d'état étant donnée généralement sous la forme $p=p(T, \bar{v})$, où $\mathrm{T}$ est la température et $\bar{v}$ le volume molaire, on définira un volume standard molaire $\bar{v}^{0}$ correspondant à un état standard de température $T$ où le gaz est parfait et où la pression standard $p^{0}$ est telle que $p^{0} \bar{v}^{0}=R T^{0}$. Un tel état standard existe toujours.

Remarque : On peut utiliser la méthode suivante pour déterminer la forme des fonctions thermodynamiques d'un gaz réel, à condition de connaître ces dernières pour le gaz parfait.

À partir de l'équation d'état, il est possible de déterminer l'énergie libre de Helmholtz en fonction de $T, \overline{\mathcal{V}}$ et les autres fonctions thermodynamiques, par une méthode approximative. Pour cela, nous admettons qu'il existe un état standard de référence défini par le volume molaire $\bar{v}^{0}$ tel que, pour $\overline{\mathcal{V}}>\bar{\nu}^{0}$, le fluide puisse être considéré comme gaz parfait, c.-à-d. $p \overline{\mathcal{V}}=R T$. Comme $\partial \bar{F} / \partial \overline{\mathcal{V}}=-p(T, \overline{\mathcal{V}})$, on peut écrire $\bar{F}=\breve{F}_{T}^{0}-\int_{\overline{\mathcal{V}}^{0}}^{\bar{\nu}} p d \mathcal{V}$. Ici apparaît la valeur standard de l'énergie libre molaire $\breve{F}_{T}^{0}$ du gaz parfait à $\overline{\mathcal{V}}=\overline{\mathcal{V}}^{0}$. On écrit $\int_{\overline{\mathcal{V}}^{0}}^{\bar{\nu}} p d \mathcal{V}=\int_{\overline{\mathcal{V}}^{0}}^{\overline{\bar{v}}}(p-R T / \overline{\mathcal{V}}) d \mathcal{V}+R T \ln \left(\overline{\mathcal{V}} / \overline{\mathcal{V}}^{0}\right) . \quad$ L'intégrale $\quad$ peut $\quad$ être écrite 
$\int_{\overline{\mathcal{V}}^{0}}^{\bar{\nu}}(p-R T / \overline{\mathcal{V}}) d \mathcal{V}=\int_{\overline{\mathcal{V}}^{0}}^{\infty}(p-R T / \overline{\mathcal{V}}) d \mathcal{V}+\int_{\infty}^{\bar{V}}(p-R T / \overline{\mathcal{V}}) d \mathcal{V} \cong \int_{\infty}^{\bar{\nu}}(p-R T / \overline{\mathcal{V}}) d \mathcal{V}$, puisque pour $\overline{\mathcal{V}}>\overline{\mathcal{V}}^{0}$, le fluide est considéré comme gaz parfait. Nous en déduisons $\bar{F} \cong \breve{F}_{T}^{0}-R T \ln \left(\overline{\mathcal{V}} / \overline{\mathcal{V}}^{0}\right)-\int_{\infty}^{\bar{v}}(p-R T / \overline{\mathcal{V}}) d \mathcal{V}$.

\subsection{Fluide de van der Waals}

L'équation d'état du fluide de van der Waals est

$$
(\bar{v}-p)\left(p+a / \bar{v}^{2}\right)=R T
$$

Cette équation d'état est la plus simple pour les gaz réels et les liquides. Elle tient compte d'une distance minimum entre les molécules et d'une interaction entre elles. Pour les gaz raréfiés : $a / \overline{\mathcal{V}}^{2}<<p$, d'où : $\overline{\mathcal{V}}-b \cong(R T / p)\left(1-a / p \overline{\mathcal{V}}^{2}\right)$ et pour les gaz parfaits (cas limite $\left.\overline{\mathcal{V}}>>b-a R T / p^{2} \overline{\mathcal{V}}^{2}\right): \bar{v}=R T / p$. Pour les liquides : $a / \overline{\mathcal{V}}^{2}>>p\left(a / \overline{\mathcal{V}}^{2}\right.$ quelques dizaines de milliers d'atmosphères), on peut souvent négliger $p$, de sorte que $\overline{\mathcal{V}} \cong \overline{\mathcal{V}}_{T}^{0}$.

Remarque : avec les variables réduites : $x=\overline{\mathcal{V}} / \overline{\mathcal{V}}_{c}, y=p / p_{c}, z=T / T_{c}$, l'équation d'état de van der Waals peut s'écrire $y=\frac{8 z}{3 x-1}-\frac{3}{x^{2}}$. En utilisant ces variables l'équation du gaz parfait devient $y_{p f t}=8 z / 3 x$.

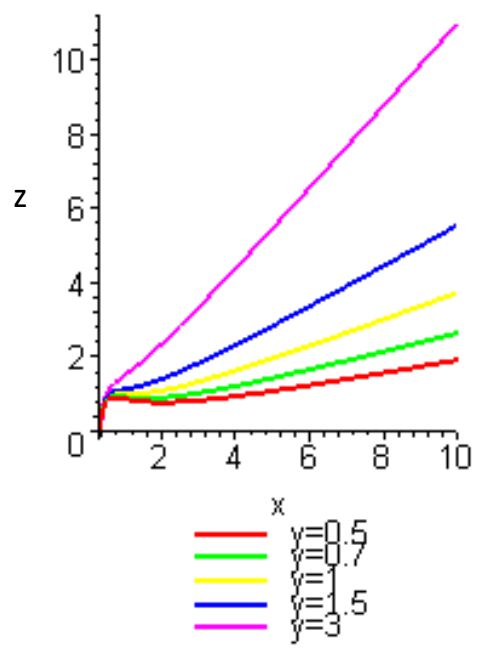

Figure 3. Définition de l'état standard : vue des isobares $z(x)$ pour $y=p / p_{c}=C t e$

Le fluide peut être considéré comme un gaz parfait si $y-y_{p f t}<<1$. Les isobares $z=(3 x-1)\left(y+3 / x^{2}\right) / 8, y=$ Cte sont représentées sur la figure 3 . On voit que ces courbes tendent à devenir des droites lorsque le volume relatif augmente. C'est la preuve que, pour un fluide de van der Waals, sur toute isotherme température donnée $T$, on peut définir un état standard pourvu que les valeurs des paramètres soient choisies dans la bonne région. Ce résultat, qui est relié à la définition d'un gaz parfait, ne dépend pas du fluide considéré.

Déterminons maintenant directement les formes de l'énergie interne $\bar{E}$, de l'entropie $\bar{S}$ et de l'enthalpie libre molaires d'un fluide de van der Waals. On peut écrire successivement ${ }^{\mathrm{v}}$ :

$p=\frac{R T}{\overline{\mathcal{V}}-b}-\frac{a}{\overline{\mathcal{V}}^{2}}, \quad d \bar{S}=\frac{1}{T} d \bar{E}+\frac{p}{T} d \overline{\mathcal{V}}=\frac{1}{T} d \bar{E}+\frac{R}{\overline{\mathcal{V}}-b} d \overline{\mathcal{V}}-\frac{a}{\mathrm{~T} \overline{\mathcal{V}}^{2}} d \overline{\mathcal{V}}=\frac{1}{T} d\left(\bar{E}+\frac{a}{\overline{\mathcal{V}}}\right)+R d \ln (\overline{\mathcal{V}}-b)$

$\bar{S}=\bar{S}\left(\bar{E}+\frac{a}{\bar{V}}, \overline{\mathcal{V}}-b\right) . \quad$ d'où $: \frac{\partial R}{\partial(\bar{E}+a / \overline{\mathcal{V}})}=0=\frac{\partial(1 / T)}{\partial \ln (\overline{\mathcal{V}}-b)}$

Il s'ensuit que, $\bar{\nu}^{0}$ correspondant à un état de référence standard, puisque $T$ n'est pas relié à $\bar{v}-b$ 


$$
\bar{E}=\bar{E}_{T}^{0}-a\left(1 / \overline{\mathcal{V}}-1 / \overline{\mathcal{V}}^{0}\right)
$$

On a $\bar{S}=\bar{S}_{T}^{0}+R \ln \left((\overline{\mathcal{V}}-b) /\left(\overline{\mathcal{V}}^{0}-b\right)\right)$, avec $\bar{S}_{T}^{0}=\bar{S}_{0}^{0}+\int_{T^{0}}^{T} d \bar{E}_{T}^{0} / T$, puisque $d \bar{S}=d\left(\bar{E}_{T}^{0} / T\right)+R d \ln (\overline{\mathcal{V}}-b)$.

Finalement est obtenue l'expression de $\bar{F}$, d'où se déduit celle de l'enthalpie libre molaire $\mu$ :

$$
\bar{F}=\breve{F}_{T}^{0}-a\left(1 / \overline{\mathcal{V}}-1 / \overline{\mathcal{V}}^{0}\right)-R T \ln \left((\overline{\mathcal{V}}-b) / \overline{\mathcal{V}}^{0}-b\right), \quad \breve{F}_{T}^{0}=\bar{E}_{T}^{0}-T \breve{S}_{T}^{0},
$$

$$
\mu=\bar{E}-T \bar{S}+p \overline{\mathcal{V}}=\breve{\mu}_{T}^{0}-2 a\left(1 / \overline{\mathcal{V}}-1 / \overline{\mathcal{V}}^{0}\right)-R T \ln \left((\overline{\mathcal{V}}-b) /\left(\overline{\mathcal{V}}^{0}-b\right)\right)+R T\left(\overline{\mathcal{V}} /(\overline{\mathcal{V}}-b)-\overline{\mathcal{V}}^{0} /\left(\overline{\mathcal{V}}^{0}-b\right)\right)
$$

Remarque : Si on applique la méthode approximative de la section 1.1, on trouve pour l'énergie libre de Helmholtz, la formule $\bar{F} \cong \breve{F}_{T}^{0}-R T \ln \left(\overline{\mathcal{V}} / \overline{\mathcal{V}}^{0}\right)-R T \ln ((\overline{\mathcal{V}}-b) / \overline{\mathcal{V}})-a(1 / \overline{\mathcal{V}})$, expression qui diffère légèrement de l'exacte [4].

\subsection{Autres lois pour les gaz et les liquides denses}

La forme suivante est applicable aux équations d'état de van der Waals, de Redlich-Kwong et de Peng-Robinson ${ }^{\mathrm{vi}, \text { vii }}$ :

$$
p=\frac{R T}{\overline{\mathcal{V}}-b} \frac{a}{\overline{\mathcal{V}}^{2}+u b \overline{\mathcal{V}}+w b^{2}}
$$

où $u$ et $w$ sont des coefficients.

Remarque : dans le cas de l'équation de Peng-Robinson

$$
u=2, w=-1, b=\frac{0,07780 R T_{c}}{p_{c}}, a=\frac{0,45724 R^{2} T_{c}^{2}}{p_{c}}\left[1+f \omega\left(1-\left(\frac{T}{T_{c}}\right)^{1 / 2}\right)\right]^{2}, f \omega=0,37464+1,54226 \omega-0,26992 \omega^{2}
$$

où le facteur acentrique $\omega$ est tabulé pour de nombreuses espèces. Ainsi : $\frac{\partial^{2} p}{\partial T^{2}}=-\frac{d^{2} a / d T^{2}}{\bar{V}^{2}+2 b \bar{V}-b^{2}}$.

Reid, Prausnitz et Poling fournissent les données nécessaires à l'obtention de l'équation d'état $p=p(T, \bar{V})$ de quelques corps, et aussi à la détermination des fonctions thermodynamiques molaires dans l'état de gaz parfait (capacités calorifiques $\bar{C}_{p, j} \mathrm{~J} / \mathrm{mol} . \mathrm{K}$, chaleurs de formation standards $\left(\bar{Q}_{f}^{0}\right)_{j}$ à 298,2 K en J/mole, et enthalpies libres de formation $\left(\mu_{0}^{0}\right)_{j}$ at 298,2 $\mathrm{K}$ sous 1 bar en J/mole).

\section{Thermodynamique des fluides au voisinage du point critique}

\subsection{Exposants universels}

En thermodynamique on considère des évolutions réversibles ou irréversibles. Pour étudier une évolution thermodynamique, on a besoin à la fois des lois de la thermostatique et de lois complémentaires faisant intervenir des coefficients de transfert. On connaît généralement ces lois loin du point critique.

Plus on s'approche du point critique, moins les lois classiques sont valables.

Ces lois classiques (par exemple celle de van der Waals) relèvent de la théorie du champ moyen. La dénomination de "Champ Moyen » provient de la propriété commune de ces modèles supposant que les interactions moléculaires n'agissent sur une molécule donnée qu'en moyenne, sans faire intervenir la position de la molécule vis-à-vis de ses voisines. C'est une hypothèse de physique statistique qui ne permet pas de prendre en compte la répartition spatiale des molécules dans l'analyse 
des interactions moléculaires. Par cette théorie on obtient des expressions analytiques faciles d'utilisation mais peu vérifiées au voisinage du point critique.

\begin{tabular}{|c|c|c|c|c|}
\hline Propriétés & $\begin{array}{c}\text { Chemin } \\
\text { thermodynamique }\end{array}$ & Expression & $\begin{array}{c}\text { Exposant de la } \\
\text { théorie du groupe } \\
\text { de renormalisation }\end{array}$ & $\begin{array}{c}\text { Exposant de la } \\
\text { théorie du } \\
\text { champ moyen }\end{array}$ \\
\hline $\begin{array}{c}\text { Compressibilité } \\
\text { isotherme }\end{array}$ & $\rho=\rho_{c}$ & $K_{T}=\Gamma\left|\frac{T-T_{c}}{T_{c}}\right|^{-\gamma}$ & $\gamma=1,239 \pm 0,002$ & $\gamma=1$ \\
\hline $\begin{array}{c}\text { Chaleur } \\
\text { spécifique } \\
\text { à volume } \\
\text { constant }\end{array}$ & $\rho=\rho_{c}$ & $C_{v}=A\left|\frac{T-T_{c}}{T_{c}}\right|^{-\alpha}$ & $\alpha=0,110 \pm 0,003$ & $\alpha=0$ \\
\hline $\begin{array}{c}\text { Saut de masse } \\
\text { volumique } \\
\text { liquide/gaz }\end{array}$ & $\begin{array}{c}\text { Courbe de } \\
\text { coexistence }\end{array}$ & $\rho_{l}-\rho_{g}=2 B\left|\frac{T-T_{c}}{T_{c}}\right|$ & $\beta=0,325 \pm 0,002$ & $\beta=0,5$ \\
\hline $\begin{array}{c}\text { Longueur de } \\
\text { corrélation }\end{array}$ & $\rho=\rho_{c}$ & $\xi=\xi_{0}\left|\frac{T-T_{c}}{T_{c}}\right|^{-v}$ & $v=0,630 \pm 0,001$ & $v=0,5$ \\
\hline Pression & $T=T_{c}$ & $p-p_{c} \propto\left(\frac{\rho-\rho_{c}}{\rho_{c}}\right)^{\delta}$ & $\delta=4,8 \pm 0,02$ & $\delta=3$ \\
\hline
\end{tabular}

Tableau 2. Quelques propriétés au voisinage du point critique et exposants critiques associés [Sengers 1978]. $\Gamma, A, B$, et $\xi_{0}$ sont les cofacteurs caractérisant la substance étudiée.

Ainsi, les conditions au point critique $\left(\frac{\partial p}{\partial \overline{\mathcal{V}}}\right)_{T}=\left(\frac{\partial^{2} p}{\partial \overline{\mathcal{V}}^{2}}\right)_{T}=0$ conduit aux relations :

$\overline{\mathcal{V}}_{c}=3 b, T_{c}=8 a / 27 R b, p_{c}=a / 27 b^{2}$, d'où l'on tire : $a=9 / 8 R T_{c} \overline{\mathcal{V}}_{c}, b=\overline{\mathcal{V}}_{c} / 3$, et aussi $: p_{c}=\frac{3}{8} \frac{R T_{c}}{\bar{V}_{v}}$. Cette dernière relation n'est pas vérifiée dans la réalité (voir le tableau 2).

La compressibilité isotherme $K_{T}$ devient dans le cas d'un fluide de van der Waals :

$$
K_{T}=-\frac{4 \overline{\mathcal{V}}_{c}^{2}}{9 R T_{c}}\left(\frac{T-T_{c}}{T_{c}}\right)^{-1}
$$

loi qui n'est pas vérifiée dans la réalité, comme le montre la première ligne du tableau 2.

En revanche, la théorie du groupe de re-normalisation permet de déduire en particulier les comportements des coefficients de transfert près du point critique. Elle fournit aussi ceux d'autres propriétés thermodynamiques.

Soit $\varphi$ une quantité physique, on considère une évolution suivant un chemin thermodynamique donné, (par exemple à masse volumique constante égale à la masse volumique critique $\rho_{c}$ ). La quantité physique $\varphi$ est alors fonction de la seule température relative $T / T_{c}$. On obtient des formules de la forme :

$$
\varphi / \varphi_{r} \propto\left(T / T_{c}-1\right)^{s}
$$


où $s$ est un coefficient constant universel, valable pour un type indiqué de transformation critique et un paramètre donné, $\varphi_{r}$ une valeur de référence pour $\varphi$.

- Si $s$ est positif la quantité considérée tend vers zéro quand la température tend vers sa valeur critique. $C^{\prime} e s t$ le cas pour la tension superficielle qui s'annule au point critique ${ }^{\text {viii, ix }}$. C'est également le cas de la compressibilité à température constante $K_{T}=(\partial \ln \rho / \partial p)_{T}$ qui peut facilement se déduire de l'équation d'état de van der Waals, comme nous l'avons vu.

Si $s$ a une valeur négative il y a une divergence au point critique. C'est le cas pour la conductivité thermique $\lambda$ qui tend ainsi vers infini. La capacité calorifique à la pression constante $c_{p}$ est également une quantité divergente.

Mais la diffusivité thermique $\kappa=\lambda / \rho c_{p}$ s'annule en approchant le point critique. D'après ce résultat, la transmission de la chaleur par conduction devient presque impossible. Un autre mode de la transmission de la chaleur a lieu qui s'appelle «l'effet de piston ».

\subsection{Evolutions isobares}

On a, à : $p=p_{c}$ selon Arias-Zugasti ${ }^{\mathrm{x}}$ :

$\rho-\rho_{c} \cong-\rho_{0}\left(\frac{T-T_{c}}{T_{c}}\right)^{1 / \delta}, \quad c_{p} \cong c_{0}\left|\frac{T-T_{c}}{T_{c}}\right|^{-\gamma / \beta \delta}, \quad \lambda \cong \rho_{c} \kappa_{0} c_{0}\left|\frac{T-T_{c}}{T_{c}}\right|^{-(\gamma-\nu) / \beta \delta}$

\subsection{Expressions valables plus ou moins loin du point critique}

On considère une évolution isobare [Arias-Zugasti 1999]. Une expression de la conductivité thermique valable plus ou moins loin du point critique est la suivante :

$\lambda=\lambda^{g a s}(T)+\lambda^{E}(\rho)+\lambda^{C}(T)$

$\lambda^{\text {gas }}(T)$ : limite à faible masse volumique, $\lambda^{E}(\rho)$ : conductivité thermique d'excès, $\lambda^{C}(T)$ : divergence critique.

- $\quad \lambda^{g a s}(T) \equiv \lambda_{\infty} T / T_{\infty}$ (Linéaire en température), ou $\lambda^{g a s}(T) \equiv \lambda_{\infty} \sqrt{T / T_{\infty}}$ (donné par la théorie cinétique des gaz).

- $\quad \lambda^{E}(\rho)=\lambda_{1} \rho / \rho_{c}$ (Dépendance linéaire en masse volumique).

- $\quad \lambda^{C}(T)=\lambda_{0}\left|\frac{T-T_{c}}{T_{c}}\right|^{-1 / 3}$ (Théorie du champ moyen).

\subsection{Point sur les approches théoriques}

Deux approches théoriques différentes sont employées couramment pour comprendre que le comportement singulier des coefficients de transport au point critique ${ }^{\mathrm{xi}}$ :

- La théorie dynamique du groupe de renormalisation évoquée plus haut, d'abord formulée par Halpering et al. est une extension des idées de la théorie statique du groupe de renormalisation de Wilson et de Fisher aux phénomènes critiques dynamiques. Ses points forts se situent dans les prévisions du comportement critique asymptotique des coefficients de transport et de l'élucidation de concepts phénoménologiques comme les échelles dynamiques et l'universalité dynamique.

- La théorie des couplages de mode, est venue de l'idée de Fixman que les anomalies critiques des coefficients de transport peuvent être comprises comme résultant de couplages non linéaires entre les modes hydrodynamiques du système. Un avantage de la théorie des couplages de mode est qu'elle peut aisément être appliquée en dehors de la région proche-critique. Partant des travaux d'Olchowy et de Gelbart, les calculs de couplages de mode et la théorie relative des modes découplés de Ferrel et de 
Perl ont été utilisés pour fournir des expressions des coefficients de transport valables en dehors de la région critique $\mathrm{xii}^{\mathrm{xii}}$.

\section{Un mode spécifique de transmission de la chaleur : l'effet piston.}

La diffusivité thermique $\kappa=\lambda / \rho c_{p}$ tend vers zéro au point critique liquide vapeur, on s'attend donc à ce que le transport de chaleur se fasse très lentement. En fait il n'en est rien, comme l'ont montré les résultats obtenus au cours d'expériences spatiales ${ }^{\mathrm{xiv}}$.

Les expériences en microgravité sont en effet nécessaires pour appréhender les phénomènes en fluide critique, car l'importante dilatabilité de ces fluides est la cause de grandes variations de masse volumique qui engendrent des mouvements convectifs intenses gênants pour l'observation précise.

En 1990, trois équipes différentes indépendantes : Zappoli ${ }^{\mathrm{xv}}$, Boukari ${ }^{\mathrm{xvi}}$, et Onuki ${ }^{\mathrm{xvii}}$, donnent l'explication de ce phénomène. Onuki y relève que l'équation de transfert de la chaleur $\frac{\partial T}{\partial t}-\kappa \nabla^{2} T=0$ n'est plus pertinente pour un fluide maintenu dans un volume fixé. Un changement

d'entropie en n'importe quel endroit dans le volume de fluide provoque un changement de pression qui à son tour engendre un changement adiabatique de température dans tout le volume. Il souligne que ce phénomène a déjà été décrit dans la littérature, mais comme il est négligeable pour un fluide à compressibilité usuelle, il a malheureusement aussi été négligé dans l'étude des fluides proches de leur point critique.

Dans un fluide incompressible, les variations d'énergie interne sont dues uniquement à des variations de température qui découlent du flux de chaleur imposé au fluide, alors que pour un fluide compressible, on doit tenir compte des variations adiabatiques de température et de densité dues aux variations de pression dans le temps [Boukari 1990]. Les effets adiabatiques sont dominants dans un fluide proche de son point critique, du fait de la divergence de sa compressibilité.

Un nouveau mécanisme de transfert de la chaleur a été mis en évidence au sein des fluides supercritiques : l'effet piston, qui agit lorsqu'on soumet une cellule remplie de fluide critique à un chauffage local.

L'équipe française ${ }^{\text {xviii }}$ [Zappoli 1990] s'est basée essentiellement sur les méthodes de perturbation singulières appliquées aux équations de Navier Stokes pour un fluide de van der Waals.

Le mécanisme de l'Effet Piston est le suivant : lorsqu'on chauffe une paroi, la chaleur diffuse depuis sa source sur une faible épaisseur, le fluide soumis à cet échauffement se dilate fortement et comprime de façon isentropique le reste du fluide, qui subit alors une augmentation homogène de sa température. En fait le fluide dilaté contenu dans la couche limite agit comme un piston, entraînant la création d'un système d'ondes de compression qui se propagent dans tout le fluide; et la réponse première du système à une perturbation d'ordre thermique sur une faible épaisseur est donc mécanique. Le passage répétitif du champ acoustique continuellement produit, engendre l'apparition d'une vitesse faible du volume de fluide ainsi qu'une compression totale de tout le volume. L'effet piston est donc le résultat d'un couplage thermomécanique représenté sur la figure 4 :

a) Le fluide supercritique confiné dans une cellule est chauffé à l'une de ces extrémités, la chaleur amenée à l'extrémité diffuse dans une couche limite thermique de faible épaisseur, dans laquelle de très forts gradients de température apparaissent (figure 4a).

b) La grande compressibilité du fluide provoquant la dilatation de la couche chaude, comprime d'une manière adiabatique le reste du fluide (figure $4 \mathrm{~b}$ ).

c) La dilatation de la couche chaude agit comme un piston sur le reste du fluide, d'où le nom de l'effet piston. On assiste à une augmentation rapide et homogène de la température dans toute la cellule (figure $4 \mathrm{c}$ ). 


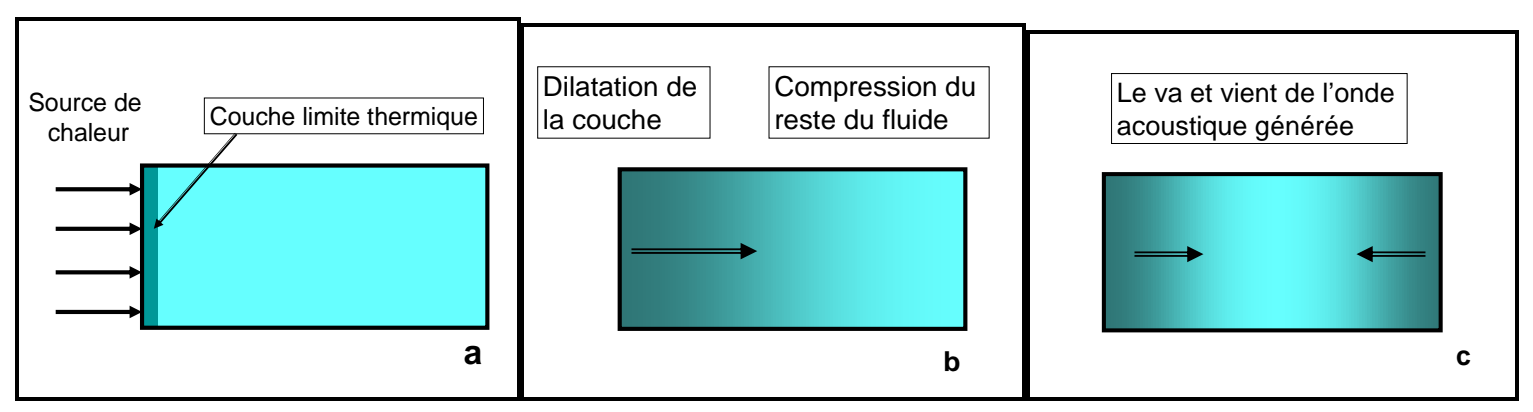

Figure 4. Représentation schématique du mécanisme de l'Effet Piston (Le niveau de foncé symbolise l'élévation de la température)

En conclusion, par un mécanisme purement dynamique, un fluide qui diffuse très mal la chaleur peut néanmoins transporter de l'énergie thermique à très grande vitesse. La relaxation de la température est donc de plus en plus rapide dans une cellule parfaitement isobare, lorsque l'on s'approche du point critique. Ce phénomène qui contraste avec le ralentissement critique de la diffusion est nommé l'accélération critique («Critical Speeding Up »).

Un gradient de température résorbé dans une cellule, entraîne des gradients de densité, qui vont à leur tour être résorbés mais cette fois sur une échelle de temps plus longue qui est de l'ordre du temps de diffusion (ou de convection si elle est présente). Le transport de chaleur a donc lieu sur l'échelle de l'Effet Piston (EP). Mais il faut attendre un temps de l'ordre du temps de diffusion pour que le fluide redevienne homogène et à l'équilibre.

Les résultats des simulations numériques présentés par Zappoli ${ }^{\mathrm{xix}}$ ont ainsi permis d'informer l'hypothèse fausse selon laquelle l'EP disparaîtrait en présence de gravité.

En effet, cette dernière étude montre que l'EP n'est pas inhibé par la convection naturelle, mais au contraire il demeure le mécanisme de relaxation de la température le plus rapide, plus rapide que la convection. Néanmoins, une autre étude expérimentale menée sur terre ${ }^{\mathrm{xx}}$ a montré qu'une cellule de fluide critique non isolée ne subissait quasiment pas d'échauffement en comparaison de ce qui se produisait en microgravité, et l'analyse des processus intervenant dans cette configuration est faite par Jounet et al. ${ }^{\mathrm{xxi}}$. Notons que l'effet piston a fait l'objet d'observations expérimentales ${ }^{\mathrm{xxii}}$ (figure5). 

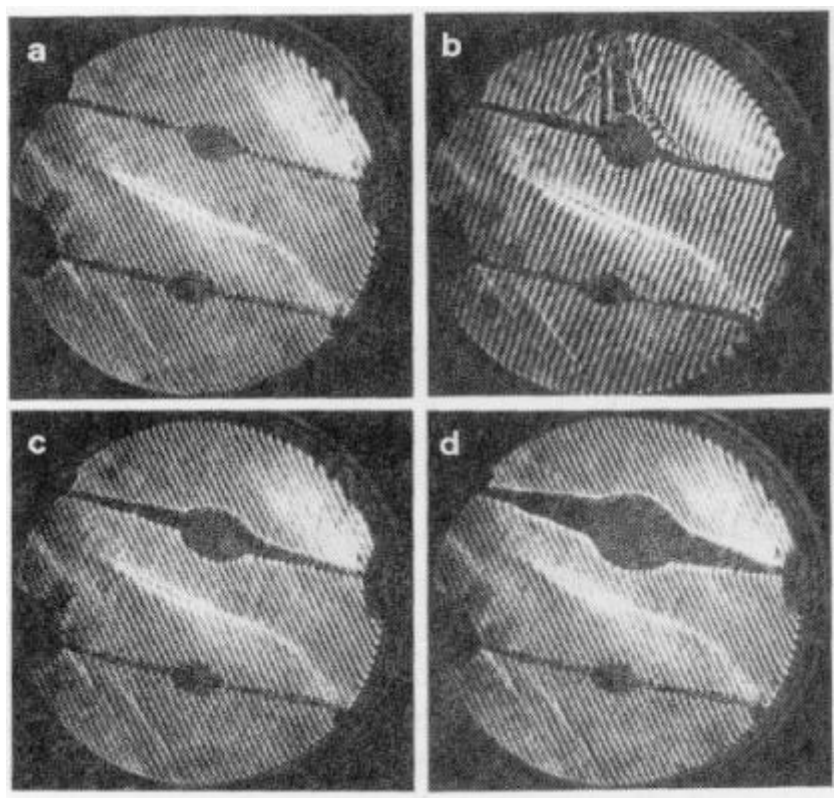

Figure 5. Evolution d'une couche limite thermique autour d'une thermistance chauffante $\left(\mathrm{CO}_{2}\right.$ à $\left.\rho=\rho_{c}\right)$.

Avant le chauffage. Vue de la cellule avec les deux thermistances. La cellule a un diamètre de $11.6 \mathrm{~mm}$. Les deux sphères sont les thermistances. Les lignes sont des franges d'interférence. (b) Expérience sous gravité terrestre ( $T=T_{c}+16 \mathrm{~K}, t=400 \mathrm{~ms}$ après démarrage du chauffage). On remarque le panache convectif. (c) Expérience en microgravité ( $T=T_{c}+16.8 \mathrm{~K}, t=400 \mathrm{~ms}$ après démarrage du chauffage). (d) Même chose qu'en (c) à $t=2 \mathrm{~s}$.

Des calculs d'écoulement ont été faits avec une cavité de rapport de forme unité, de longueur $\mathrm{L}=10$ $\mathrm{mm}$, remplie de $\mathrm{CO}_{2}$ supercritique. La paroi inférieure est chauffée, linéairement en fonction du temps, de $10 \mathrm{mK}$ pendant une seconde. Le fluide est initialement à $1 \mathrm{~K}$ du point critique.

La figure 6 donne le champ de température dans des conditions précisées. On observe des panaches thermiques près des parois chaude et froide. La couche supérieure devient instable, témoignant ainsi de l'existence d'un effet piston froid, avec une couche limite de contraction à la paroi supérieure placée à la température initiale. Entre les parois, la température est assez homogène ${ }^{\text {xxiii }}$.

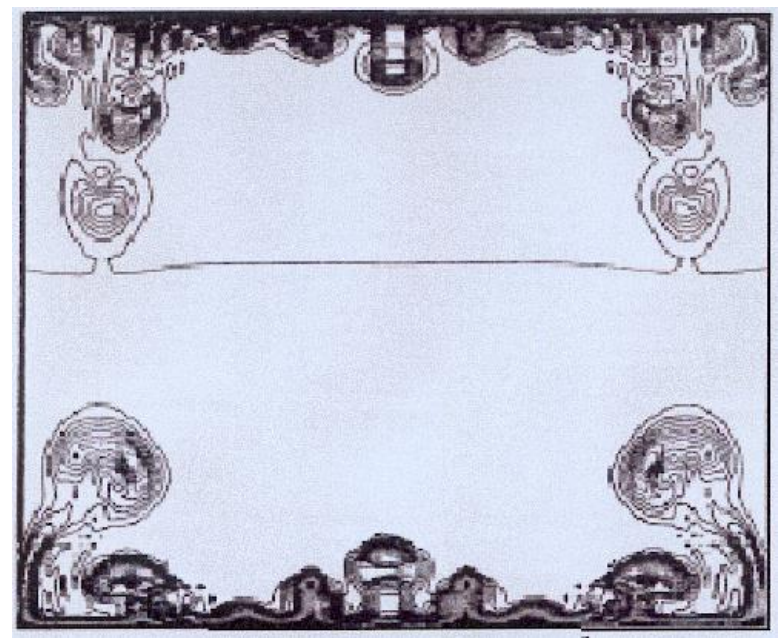

Figure 6. Champ de température à $t=8.8 \mathrm{~s}$ avec une différence de température entre les deux parois de $\Delta T=10 \mathrm{mK}$ à $1 \mathrm{~K}$ du point critique. 


\section{Expansion d'une "goutte" a la pression critique}

Aux pressions supercritiques, dans le cas de corps purs, il n'y a pas de phases distinctes de liquide et de vapeur : le domaine entier est monophasique. La relaxation de densité d'une poche liquide dense dans une atmosphère plus chaude et isobare est néanmoins semblable au processus de vaporisation d'une gouttelette liquide sous critique à condition que la diffusivité thermique de la poche liquide soit plus faible que celle de l'atmosphère environnante. Une zone où les gradients de densité et de temps de diffusion sont importants isole la poche dense de l'atmosphère environnante ${ }^{\mathrm{xxiv}, \mathrm{xxv}}$. La poche dense est ainsi appelée «gouttelette» supercritique et la zone de fort gradient de densité est comparée à une « interface » épaisse.

Pour une poche liquide critique (densité critique, température critique) introduite dans une atmosphère (plus chaude) à la pression critique, la condition de diffusivité précédemment mentionnée est valable pour toutes les températures de l'atmosphère en raison de la divergence critique de la diffusivité dans la poche critique. Aux pressions clairement supercritiques, cet état de diffusivité est plus restrictif et nécessite, en condition de fluide idéal, $T_{\infty}>>T_{i}$, avec $T_{\infty}$ température de l'atmosphère et $T_{i}$ température initiale de la poche liquide dense. L'« interface » étant épaisse, deux limites peuvent être choisies pour la définition de la frontière de référence de la «gouttelette » supercritique : la première consiste à choisir une isochore $\rho_{m}$ localisée à la fin de la zone de fort gradient de densité de la poche fluide. On peut constater que cette surface située à la fin de la zone de fort gradient de densité est le lieu où le débit masse unitaire est maximum $r=r_{m}$. La seconde consiste en l'isochore de référence $\rho_{0}$ au début de la zone de fort gradient de densité $\mathrm{r}=\mathrm{r}_{0}$. Entre ces deux limites (zone de transition), on rapporte les distances à l'épaisseur de cette «interface »: $X=\left(r-r_{0}\right) /\left(r_{m^{-}}-r_{0}\right)$ et on considère que l'évolution est quasi stationnaire avec cette nouvelle abscisse. Pour $r<r_{0}$ (zone intérieure) les paramètres sont constants. Pour $r>r_{m}$, l'évolution est quasi stationnaire dans les coordonnées naturelles. Ces considérations permettent une résolution analytique. Appelons $D_{m}$ le diamètre du domaine limité par l'isochore $\rho_{m}$ et $D_{0}$ le diamètre du domaine limité par l'isochore $\rho_{0}$.

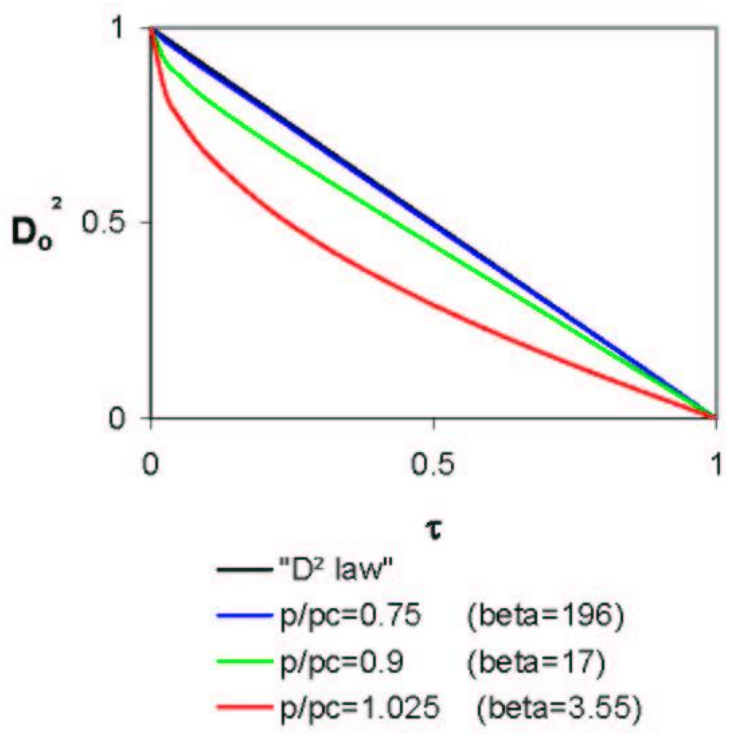

Figure 7. Carré du diamètre de la gouttelette en fonction du temps réduit $\tau$ pour différentes pressions. $p_{c}$ est la pression critique.

Il est démontré que le diamètre $D_{m}$ vérifie la loi en " $\mathrm{D}^{2}$ " : $D_{m}^{2}=1-\tau$, avec $\tau$ temps adimensionnel, rapport entre le temps et le temps de vie de la goutte. Le diamètre $D_{0}$ vérifie (voir figure 7) : 


$$
D_{o}^{\beta}-\beta D_{o}=(1-\beta) \sqrt{1-\tau}
$$

Le paramètre $\beta$ est de l'ordre de l'unité aux pressions légèrement subcritique ou supercritique : la loi en $D^{2}$ n'est pas vérifiée pour $D_{0}$ à de telles pressions comme le montre la relation précédente. Lorsque la pression devient nettement subcritique, $\beta$ devient grand.

Puisque le diamètre $D_{o}$ est dimensionné par le diamètre initial de la gouttelette, $D_{o}(\tau) \leq 1$ et, pour les grandes valeurs de $\beta$, l'équation précédente devient au premier ordre : $D_{o}^{2}=1-\tau$. La loi en $D^{2}$ est ainsi retrouvée aux pressions nettement subcritiques.

La résolution numérique basée sur une méthode de discrétisation de type volumes finis pour une géométrie à symétrie sphérique utilise l'algorithme SIMPLER pour la résolution des équations filtrées du système. Les résultats de la résolution analytique sont en très bon accord avec ceux obtenus numériquement.

\section{Remarques :}

- Il serait intéressant de définir une tension superficielle effective en conditions supercritiques. En effet, les phénomènes analogues à ceux observés en fluides miscibles menant à la tension superficielle effective, apparaissent également en fluide pur quand les gradients de température élevés se produisent ${ }^{\mathrm{xxvi}}$. Ceci est illustré (figure 8) dans le cas d'une goutte froide tombant dans le même fluide plus chaud ${ }^{\mathrm{xxvii}}$.

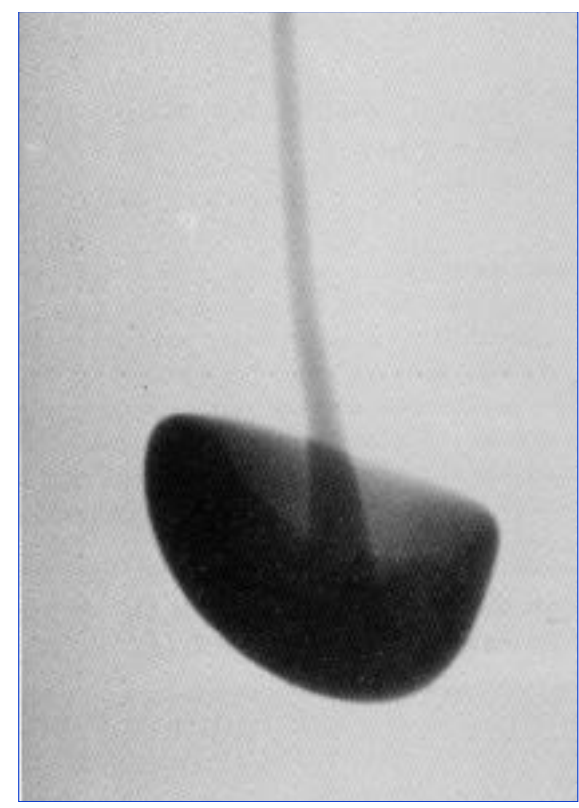

Figure 8. Une goutte thermique. La goutte froide à $-7^{\circ} \mathrm{C}$ tombant dans le même liquide initialement à $21^{\circ} \mathrm{C}$ [Kojima et al. 1984]

- Un problème légèrement différent est mis en jeu en particulier dans la zone d'injection des moteurs cryogéniques. On doit considérer la vaporisation isobare à la pression supercritique d'une « gouttelette » froide dans une ambiance chaude. La gouttelette est au début à une température sub-critique. Hors de la zone prochecritique il n'y a aucune singularité de la conductivité thermique, et il apparaît donc une période d'échauffement de la goutte, comme cela se produit dans le cas d'un liquide plongé dans un gaz parfait, mais ici avec une équation d'état de gaz réel. 


\section{Comportement d'une poche de fluide supercritique plongée dans un environnement a haute température}

Le cas d'une goutte de fluide pur à l'état critique plongée dans une ambiance isobare de densité plus faible, qui a fait l'objet de la thèse de $\mathrm{S}$. Préau au $\mathrm{LMM}^{2}$, a permis d'établir analytiquement et numériquement la loi d'évaporation; les conséquences de cette loi restent à explorer et d'autres cas d'évaporation où la goutte initiale est à la pression critique mais à une température inférieure à la température critique restent à étudier.

Dans certains foyers de combustion, des gouttes liquides (de combustible ou de comburant) sont injectées dans une atmosphère très chaude et à pression supérieure à la pression critique du liquide considéré.

Ces gouttes apparaissent comme des poches fluides en mouvement et en expansion, sans interface de changement de phase.

Le cas où la goutte initialement sphérique se trouve dans les conditions critiques et s'épand de façon isobare, en restant sphérique, dans le même fluide au repos de température à l'infini très supérieure à la température critique, a été étudié [S. Préau et al., 2004].
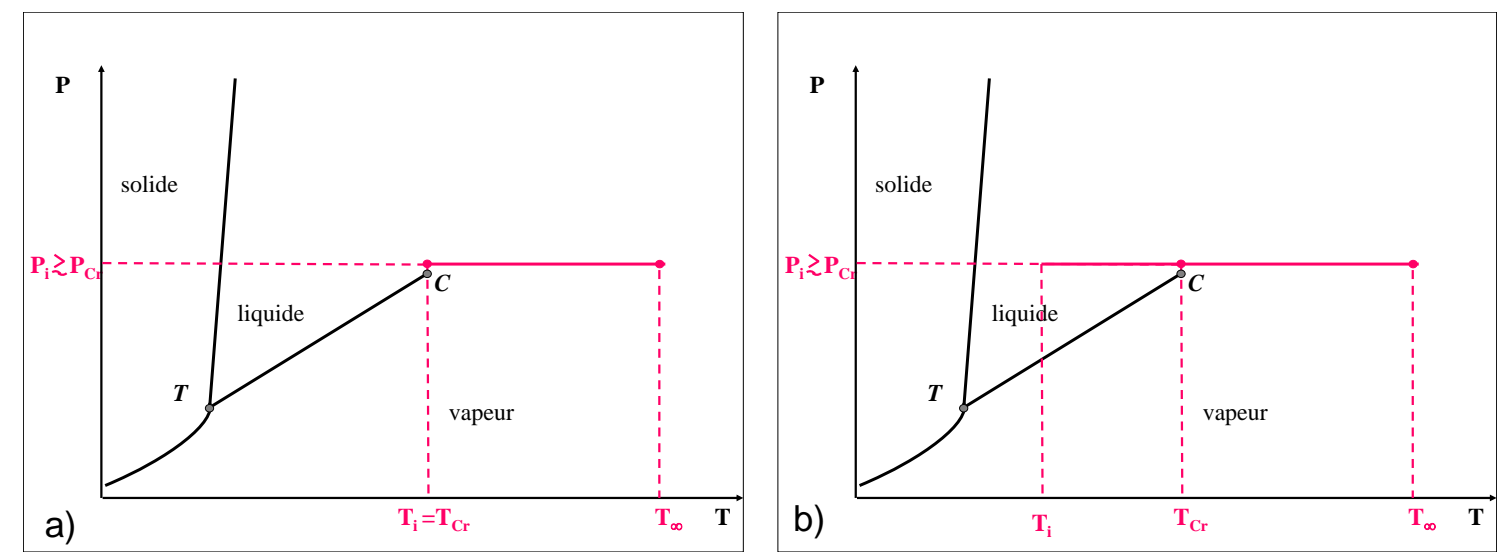

Figure 9. Diagramme température-pression d'un corps pur, $T:$ point triple, $C:$ point critique, $\left(T_{i}, p_{i}\right):$ conditions initiales, $\left(T_{\infty}, p_{i}\right)$ : conditions finales. a) Goutte proche critique à l'état initial [Préau 2004] ; b) Goutte froide à l'état initial [SAGNA 2014]

On se propose d'étendre ici l'étude au cas où la température initiale de la goutte est inférieure à la température critique et où la pression ambiante est supérieure à la pression critique (figure 9).

Cette situation est assez représentative de moteurs cryogéniques (fusée Ariane par exemple) et de moteurs Diesel.

Dans la réalité, l'existence d'un écoulement turbulent extérieur et l'absence de tension superficielle conduisent à une rupture de la symétrie sphérique et à l'épluchage de la poche.

Néanmoins on supposera ici que la poche reste sphérique, pour simplifier mais aussi pour établir des lois d'évolution ${ }^{\text {xxviii }}$ qui pourront être introduites dans des modèles plus complexes incluant la turbulence. La partie numérique de l'étude peut se faire à l'aide d'un code existant fourni par Jalil Ouazzani. Pour la partie analytique, la méthode des développements asymptotiques est préconisée. Une étude analytique et numérique a été menée dans ce sens par Sagna ${ }^{\text {xxix }}$ en faisant des hypothèses supplémentaires sur les propriétés thermodynamiques de la phase gazeuse pour restaurer la loi en $D^{2}$ classique $^{\mathrm{xxx}}$, ce qui n'est pas possible dans le cas critique conduisant à l'équation [10]. 


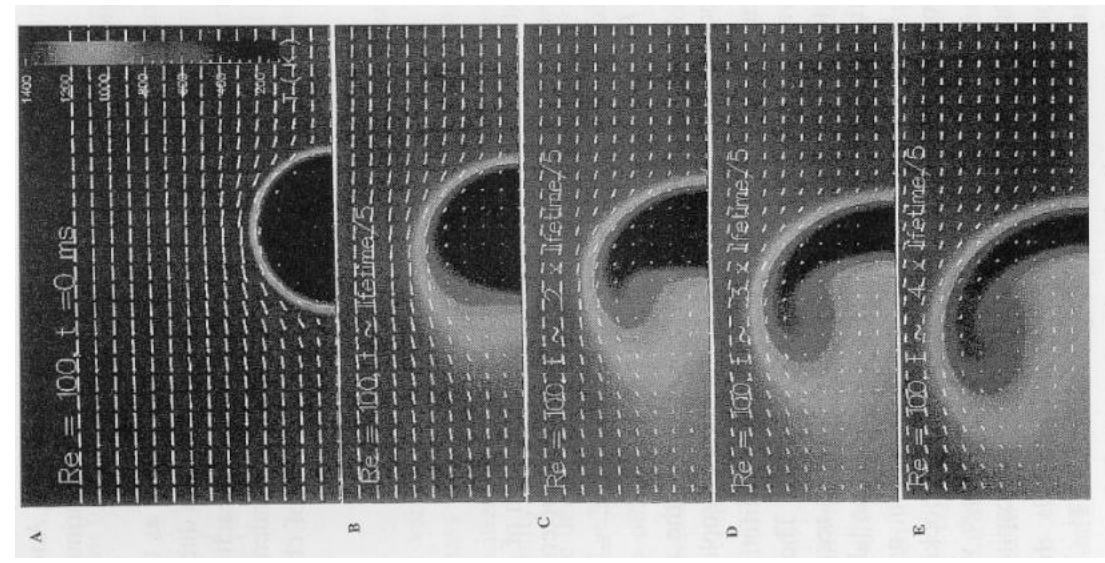

Figure 10. Le chauffage et la déformation d'une poche dense pendant sa durée de vie. La gamme de teintes est relative au champ de température. Nombre de Reynolds d'injection : $R e=100$, nombre de Prandt : $\operatorname{Pr}=1$, et rapport de densité $=15$. Température dimensionnelle d'injection : $100 \mathrm{~K}$, température ambiante $: T_{\infty}=1500$ $K$, rayon initial de la poche $: a_{o}=50 \mu m$ [Kojima et al. 1984].

Notons que le problème du chauffage de poches de gaz denses dans les écoulements a déjà fait l'objet d'études numériques. La figure 10 montre les déformations d'une telle poche.

\section{Ebullition près du point critique ${ }^{3}$}

Des expériences de croissance de bulle seront effectuée avec l'instrument DECLIC ${ }^{4}$ dans la station Spatiale Internationale. L'instrument DECLIC est voué à l'étude de la solidification en milieu transparent et à celle des fluides critiques.

Remarque : Un avantage de mener des expériences près du point critique réside dans la relative lenteur des phénomènes, ce qui facilite notablement les observations.

L'hyper compressibilité des fluides dans la région critique permet un transport adiabatique extrêmement rapide de la chaleur, que nous avons mis en évidence en apesanteur [Garrabos 1998] et baptisé « effet Piston »: la couche limite thermique près de la paroi chauffante se dilate et comprime et chauffe adiabatiquement le reste du fluide à la vitesse du son. De nouvelles expérimentations dans l'équipement ALICE2 dans la station MIR ont été réalisées, qui montrent que ce mécanisme de transport de la chaleur peut conduire à des résultats paradoxaux ${ }^{\mathrm{xxi}}$, violant apparemment, comme cela a été cité ${ }^{\mathrm{xxxii}}$ [Sincell 2000], le deuxième principe de la thermodynamique : quand les deux phases gaz et liquide d'un fluide $\left(\mathrm{SF}_{6}\right)$ sont chauffées en apesanteur, le gaz peut en effet devenir plus chaud que la paroi chauffante!

Cette surchauffe est maximum à $5^{\circ} \mathrm{C}$ du point critique où elle est supérieure à $20 \%$ (figure 10 ). Ce paradoxe peut être levé si l'on considère qu'il y a transfert thermique et transfert mécanique : la couche de liquide, dilatée par la chauffe du corps de la cellule, comprime plus la vapeur que le liquide. La nature thermo-compressible de l'effet Piston permet donc d'expliquer cette apparente violation.

Cette anomalie n'est visible que si la bulle de gaz est éloignée de la paroi de la cellule. Si ce n'est pas le cas, d'autres phénomènes sont observés, en relation avec la crise d'ébullition familière aux thermiciens, quand le flux de chaleur est si fort que le liquide s'évapore et forme un film de vapeur à la paroi, empêchant le transfert de chaleur.

\footnotetext{
${ }^{3}$ D'après Yves Garrabos, CNRS Talence et Daniel Beysens, CEA/ESPCI Paris

${ }^{4}$ DECLIC : Dispositif d'Étude de la Croissance et des Liquides Critiques.
} 


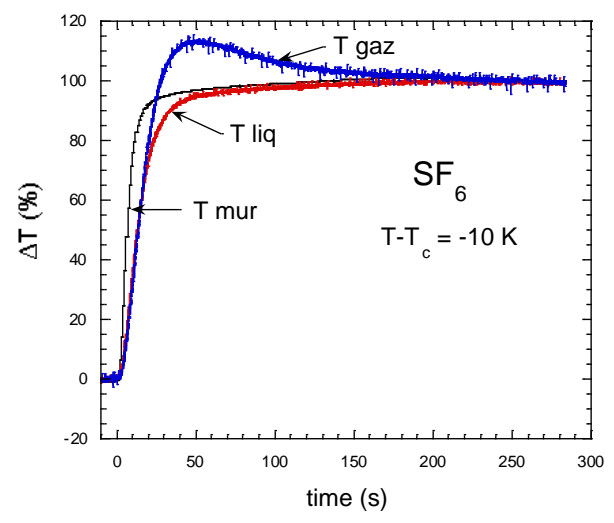

Figure 11. Surchauffe observée lors d'une trempe thermique de $0,1^{\circ} \mathrm{C}$ dans une cellule de SF6 initialement à $10^{\circ} \mathrm{C}$ sous son point critique : la température du gaz est supérieure à la paroi chauffante.

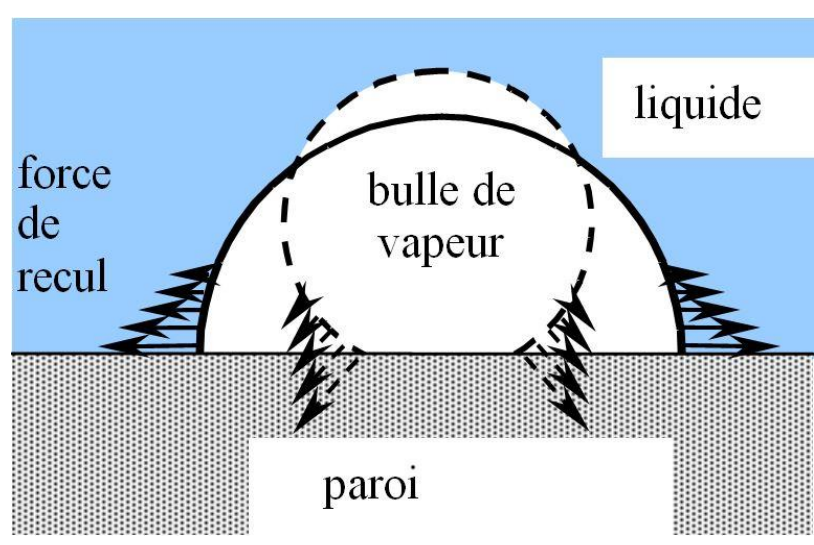

Figure 12. Etalement de la bulle de vapeur sur une paroi chauffante sous l'action de la force de recul de la vapeur.

Ce phénomène a été interprété ${ }^{x x i i i}$ par la dynamique de la ligne triple solide-liquide-vapeur, où la poussée de l'évaporation produit une force - la force de recul - qui tire la ligne de contact vers la phase liquide et étale la vapeur sur la paroi (figure 11). Dans une cellule où maintenant la bulle de gaz est comprimée par les parois on observe un étalement de gaz à la paroi ${ }^{\text {xxxiv }}$ (figure 12). C'est une véritable crise d'ébullition, visible ici à faible flux thermique parce que l'absence de gravité et la proximité du point critique rendent négligeables les forces de pesanteur et la chaleur latente de vaporisation. Cette transition par tirage de ligne de contact est la démonstration expérimentale des idées développées dans des études théoriques et numériques ${ }^{\mathrm{xxv}}$, où nous il a été proposé ce mécanisme pour rendre compte de la crise d'ébullition (figure 13).
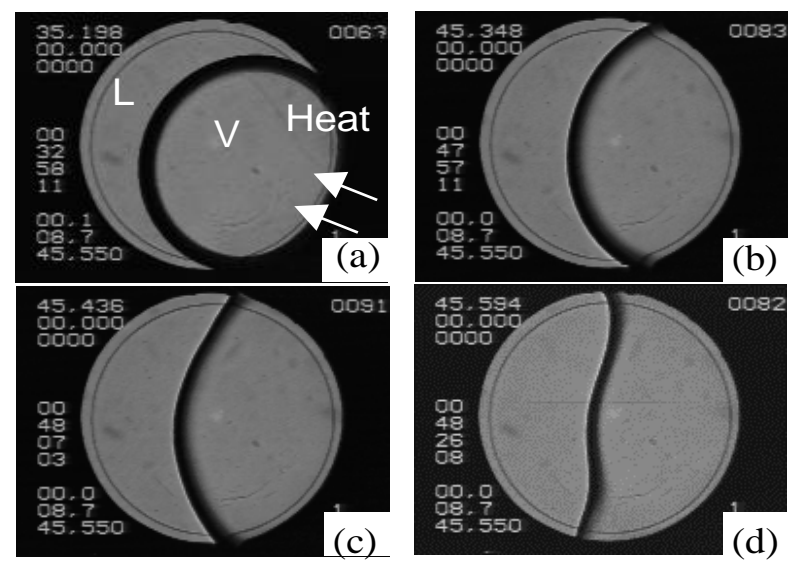

(e)

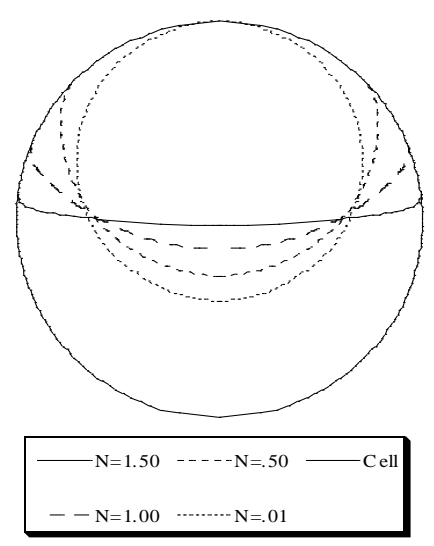

Figure 13. Déformation de l'interface liquide - vapeur sous l'action de la force de recul. (a-d) : Fluide $S F_{6}$ en apesanteur soumis à un flux de chaleur. (a) : $T-T_{c}=10.4 \mathrm{~K}$, (b) $T-T_{c}=0.25 \mathrm{~K}$, (c) $T-T_{c}=0.15 \mathrm{~K}$, (d) $T-T_{c} \approx 0$. (e) : simulation $2 D$ : Le paramètre $N$ varie comme la différence $\left(T-T_{c}\right) / T_{c}$. Fraction volumique de la vapeur : 0.5. L'angle de contact réel est zéro.

Une mise au point sur ces questions liées à la micropesanteur a été publiée récemment ${ }^{\mathrm{xxxvi}}$. 


\section{Conclusion}

Si cet article débute par un rappel sur la thermodynamique des fluides critiques et supercritiques, c'est que les propriétés de ces fluides sont très particulières. Leurs lois de comportement présentent en effet des divergences au point critique.

Chaque cas d'interface évoqué est influencé par ces lois de comportement : qu'il s'agisse de l'effet piston où l'on a affaire au transfert de chaleur à la paroi, de l'expansion d'une goutte à la pression critique où les phénomènes se passent à la surface de la goutte, ou encore de l'ébullition avec l'action du recul de la vapeur près de la ligne de contact avec la paroi.

Le voisinage du point critique a un effet majeur sur les phénomènes d'interface dont l'analyse mathématique, la modélisation numérique et l'observation expérimentale présentent des spécificités, comme nous avons pu le constater.

Sur le plan expérimental le recours à la micropesanteur a été systématique et la qualité exigée (en finesse et en durée) pour cette micropesanteur a conduit à choisir l'expérimentation en satellite avec l'appui de diagnostics évolués. C'est donc à un effort particulier dans l'intensité et la durée que l'on doit les résultats obtenus. Cela n'a été possible qu'avec un accompagnement structurel, scientifique et financier du CNES, du CEA, du CNRS et de laboratoires universitaires. La coopération internationale a joué tant pour les lanceurs que pour les satellites et sur le plan scientifique.

\section{Références}

i Zappoli B., Beysens D., Garrabos Y. (2015) Heat Transfers and Related Effects in Supercritical Fluids. Fluid Mechanics and Its Applications. Volume 108 Springer. ISBN: 978-94-017-9186-1 (Print), 978-94-017-9187-8 (Online).

ii Beysens D.: Near-critical fluids under micro gravity: Highlights and perspectives for Europe. J. Phys. IV France, 11 (2001) Pr6-7-22

${ }^{\text {iii }}$ B. Zappoli, D. Bailly, Y. Garrabos, B. Le Neindre, P. Guenoun, D. Beysens, Anomalous heat transport by the piston effect in supercritical fluids under zero gravity, Phys. Rev. A 41 (4) (1990) 2264-2267.

${ }^{\text {iv }}$ Garrabos Y., Le Neindre B., Subra P., Cansel F., Pommier C. : Fluides critiques et gravité, fluides supercritiques et matériaux. In Fluides, Matériaux et Microgravité, Ann. Chim., FR., 17, 55-90, 1992.

${ }^{v}$ Prud'homme R. : Fluides hétérogènes et réactifs : écoulements et transferts, Lecture Notes in Physics, 304, Springer Verlag, 1988.

${ }^{\text {vi }}$ Prausnitz, J., Rüdiger, M., Lichtenthaler, N., Gomes de Azevedo, E.: Molecular thermodynamics of fluid-phase equilibria, Third edition, Prentice Hall PTR, Upper Saddle River, New Jersey, 1999.

${ }^{v i i}$ Reid, R.C., Prausnitz, J.M., Poling, B.E.: The properties of gases and liquids, Fourth edition, Mc Graw Hill, Boston (1987). OSTI ID: 6504847

viii Eddy Mezger. Loi de variation de la tension superficielle avec la température. J. Phys. Radium, 1946, 7 (10), pp.303309. <10.1051/jphysrad:01946007010030300>. <jpa-00233998>

${ }^{\text {ix }}$ C. Bachaud. La variation de la tension superficielle près du point critique. Rôle des densités du liquide et de la vapeur saturante. J. Phys. Radium, 1951, 12 (8), pp.825-826. <10.1051/jphysrad: 01951001208082501>. <jpa-00234488>

${ }^{x}$ Arias-Zugasti M., Garcia-Ibarra P.L., Castillo J.L.: Droplet vaporization at critical conditions: Long-time convectivediffusive profiles along the critical isobar, Physical Review E, 60, №3, 2930-2941, 1999.

${ }^{x i}$ Luettmer-Strathmann, L., Sengers, J. V., Olchowi, G. A.: Non-asymptotic critical behavior of the transport properties of fluids, J. Chem. Phys. 103 (17), 7482-7501 (1995).

${ }^{x i i}$ Sengers JV, Levelt Sengers JMH: Critical phenomena in classical fluids, in Progress in liquid Physics, Ed. Croxton C., Wiley, Chichester (UK), 1978.

xiii Sengers, J.V.: International Journal of Thermophysics, 6, 203 (1985). 
${ }^{\text {xiv }}$ Nitsche K. and Straub J.: The critical "hump" of $C_{v}$ under micro gravity, result from the D-1 Spacelab experiment 'Wärmekapazität", Proceeding of 6th European Symposium on Material Science under Microgravity Conditions, ESA SP-256, Bordeaux France, 1987.

${ }^{\mathrm{xv}}$ Zappoli B., Bailly D., Garrabos Y., Le Neindre B., Guenoun P. and Beysens D.: Anomalous heat transport by the piston effect in supercritical fluids under zero gravity. Physical Review A, 41(4): 2264-2267, 1990.

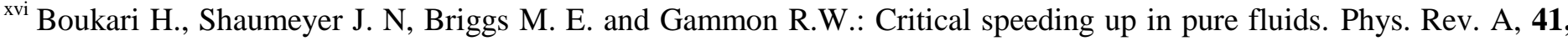
2260, 1990. https://doi.org/10.1103/PhysRevA.41.2260

xvii Onuki A., Hong H. and Ferreli R.A.: Past adiabatic equilibration in a single component fluid near the liquid-vapor critical point, Phys. Rev. A, 41:2256, 1990. https://doi.org/10.1103/PhysRevA.41.2256

xviii Prud'homme R.: Bernard Zappoli's contribution to the French scientific life. CR Mecanique (2016), http://dx.doi.org/10.1016/j.crme.2016.10.006

${ }^{\text {xix }}$ Zappoli B., Amiroudine S., Carlès P., Ouazzani J.: Thermo acoustic and bouncy-driven transport in a square sideheated cavity filled with a near-critical fluid, J. Fluid Mech., 316, 53-72, 1996.

${ }^{x x}$ Beysens D. : Que peut-on apprendre sur les phénomènes de transport d'une étude en microgravité. Ann. Chim. Fr., 17, 45-53, 1992.

${ }^{x x i}$ Jounet A., Zappoli B. Mojtabi A.: Simulation numérique de l'effet Piston avec prise en compte des échanges thermiques pariétaux. C.R.A.S. Paris, 327, série IIb., pp. 991-996, 1999.

${ }^{x x i i}$ Bonetti M., Perrot F., Beysens D.: Fast thermalization in supercritical fluids, Physical Review, 49, N $6,1994$. https://doi.org/10.1103/PhysRevE.49.R4779

xxiii Amiroudine S., Bontoux P., Laroudé P., Gilly B., Zappoli B.: Direct numerical simulation of instabilities in a twodimensional near-critical fluid layer heated from bellow, J. Fluid Mech., 442, 119, 2001.

${ }^{x x i v}$ Sanchez-Tarifa C., Crespo A., Fraga E.A., 1972, "Theoretical Model for the Combustion of Droplets in Super-critical Conditions and Gas Pockets", Astron. Acta, 17, pp. 685-692.

${ }^{x x v}$ Préau S., Prud'homme R., Ouazzani J., Zappoli B., 2004, "Supercritical density relaxation as a new approach of droplet vaporization", Phys. Fluids, 16, pp 4075-4087. https://doi-org.accesdistant.sorbonneuniversite.fr/10.1063/1.1790231

${ }^{x x v i}$ Gatignol, R. \& Prud'homme, R., 2001, Mechanical and thermodynamical modeling of fluid interfaces- Series on Advances in Mathematics for Applied Sciences, 58, World Sientific, Singapore. http://www.worldscibooks.com/mathematics/4422.html

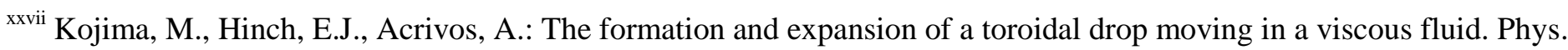
Fluids, 27 (1), 19-32 (1984). https://doi.org/10.1063/1.864511

xxviii D'Almeida A., Prud'homme R., Evaporation de gouttes lois de récession du rayon, 19ème Congrès Français de Mécanique - Marseille, 24-28 août 2009.

${ }^{x x i x}$ Sagna K., D’Almeida A., A Study of Droplet Evaporation. American Journal of Modern Physics. Vol. 2, No. 2, 2013, pp. 71-76. doi: 10.11648/j.ajmp.20130202.17

${ }^{x x x}$ Sagna K., D'Almeida A., Supercritical Evaporation of a Drop. International Journal of Research in Engineering and Science (IJRES) ISSN (Online): 2320-9364, ISSN (Print): 2320-9356 www.ijres.org, Vol. 2 Issue 7 (July. 2014) pp. $41-47$

${ }^{x x x i}$ Wunenburger R., Garrabos Y., Chabot C., Beysens D., Hegseth J. Thermalization of a two-phase fluid in low gravity: heat transfer from cold to hot. Phys. Rev. Lett. 2000, 84, 4100-4103. https://doi.org/10.1103/PhysRevLett.84.4100

xxxii Sincell M. Backward heat flow bends the law a bit. Science 2000, 288, 789-791.

xxxiii Nikolayev V., Beysens D. Boiling crisis and nonequilibrium wetting transition. Europhys. Lett. 1999, 47, 345-351.

${ }^{\text {xxxiv }}$ Garrabos Y., Lecoutre-Chabot C., Hegseth J., Nikolayev V., Beysens D., Delville J.-P. Gas spreading on a heated wall wetted by liquid Phys. Rev. E 2001, 64, 051602, 1-10. https://doi.org/10.1103/PhysRevE.64.051602

${ }^{x x x v}$ Nikolayev V., Beysens D., Lagier G.-L., Hegseth J. Growth of a dry spot under a vapor bubble at high heat flux and high pressure. Int. J. Heat \& Mass Transfer 2000, vol. 44/18, 3499-3511. https://doi.org/10.1016/S00179310(01)00024-2

${ }^{x x x v i}$ V. S. Nikolayev et al., Evaporation condensation - induced bubble motion after temperature gradient set up. CR Mecanique 2016 http://dx.doi.org/10.1016/j.crme.2016.10.004 